\title{
Interaction Potential for InSb: A Molecular Dynamics Study
}

\author{
J. P. Rino, P. S. Pizani, and S. C. Costa \\ Departamento de Física, Universidade Federal de São Carlos, \\ Caixa Postal676, 13565-905, São Carlos, SP, Brazil
}

Received on 6 September, 2003

\begin{abstract}
Molecular dynamics simulation was used to study structural and dynamical properties of InSb. The effective potential takes into account two and three-body interactions, considering atomic-size effects and charge-charge, charge-dipole, and dipole-dipole interactions between 1000 particles, $500 \mathrm{In}$ and $500 \mathrm{Sb}$, initially within a cubic box of side $L=32.397 \AA$. The effect of hydrostatic pressure and temperature on the structural properties like pair distribution function, coordination number, volume change and bond angle distribution and on dynamical properties like vibrational density of states, phonon anharmonicity, dynamic Debye-Waller factor, thermal expansion coefficient and structural phase transformations are correctly described, in excellent agreement with the experimental results.
\end{abstract}

\section{Introduction}

Since the eighties molecular dynamic (MD) calculations can simulate structural phase transformations because of the modifications introduced by Parrinello and Rahman [1] who used an appropriate Lagrangian that permits MD calculations in which both the volume and the shape of the simulation box change with time. This is so worth because many materials exhibit crystal phase changes under temperature and pressure. In particular, many of the III-V semiconductors undergo a semiconductor to metal transition under high pressure. Among then, InSb is one that present the lowest pressure induced structural transformation.

In the seventies x-ray diffraction was used to study the structural transformation in indium antimonide up to 2.8 $\mathrm{GPa}[2,3,5]$. In next decade Vanderborgh et al., using energy-dispersive $\mathrm{x}$-ray diffraction studied this system up to $66 \mathrm{GPa}[6]$. The most of the experimental results indicated that its cubic structure, at room temperature, transforms either to a mixture of $\mathrm{P} 2$ (tetragonal $\beta$-tin structure) and $\mathrm{P} 3$ (orthorhombic) phases at $\sim 2.1 \mathrm{GPa}$, followed to a singlephase $\mathrm{P} 3$ and before crystallizing in $\mathrm{P} 4$ orthorhombic phase, or directly transform to P4 at $\sim 3.0 \mathrm{GPa}$. Recently, Nelmes et. al.[7] reexamined, by using angle-dispersive powderdiffraction technique on a synchrotron source, the structural transformation in InSb up to $5 \mathrm{GPa}$, showing that the established pressure-temperature (P-T) phase diagram was incorrect. Finally, further experiments[8] has shown that the phase P2, in fact, does not exist, but is an orthorhombic phase. On the other hand, from the theoretical point of view, there are some pseudo-potential and first-principle density functional total energy calculations studies about this mate- rial. However, these calculations are performed at structural ground state configuration [9-11].

In this paper we report the results of isoenthalpicisobaric MD simulation for the pressure induced structural transformation and dynamical properties in InSb. From the MD simulation, dynamical Debye-Waller factor, thermal expansion coefficient, pressure induced phase transition, temperature and pressure phonon anharmonicity were correctly described, the last in excellent agreement with Raman scattering experimental results.

\section{Interaction potential and molecular dynamics calculation}

The central core of a molecular dynamics simulation is the choice of the interatomic potential, which determines the failure or success of a simulation. There are tens of empirical interaction potentials, which have been used to describe elemental semiconductors and metals to III-V and II-VI semiconductors or more complex systems [12, 13]. Among all these empirical interaction potentials we choose the interaction potential proposed by Shimojo et. al.[14] which has been used to describe several different systems [15-18]. The total interaction potential consist of effective two-body and three-body terms

$$
\Phi=\sum_{i \prec j} V_{i j}^{(2)}\left(r_{i j}\right)+\sum_{i \prec j \prec k} V_{j i k}^{(3)}\left(r_{i j}, r_{i k}\right) .
$$

The two-body interaction reads as

$$
V_{i j}^{(2)}\left(r_{i j}\right)=\frac{H_{i j}}{r_{i j}^{\eta_{i j}}}+\frac{Z_{i} Z_{j}}{r_{i j}} \exp \left(-r_{i j} / \lambda\right)-\frac{D_{i j}}{2 r_{i j}^{4}} \exp \left(-r_{i j} / \xi\right)-\frac{W_{i j}}{r_{i j}^{6}}
$$


where the first term takes into account stereometric repulsion (with parameters $H_{i j}$ and $\eta_{i j}$ ), the second term is the Coulomb interaction due to charge transfer between ions, the third term is the charge-dipole interaction due to the large electronic polarizability of anions, and the last one is the van der Waals (dipole-dipole) type interaction. The three-body interaction, necessary to take into account covalent effects, is a modified Stillinger-Weber [12] type potential given by

$$
V_{j i k}^{(3)}\left(r_{i j} r_{i k}\right)=B_{i j k} \exp \left[\frac{\gamma}{r_{i j}-r_{0}}+\frac{\gamma}{r_{i k}-r_{0}}\right] \frac{\left(\cos \theta_{i j k}-\cos \left\langle\theta_{i j k}\right\rangle\right)^{2}}{1+C\left(\cos \theta_{i j k}-\cos \left\langle\theta_{i j k}\right\rangle\right)^{2}} \Theta\left(r_{0}-r_{i j}\right) \Theta\left(r_{0}-r_{i k}\right),
$$

where $B_{i j k}$ is the strength of the interaction, $\Theta\left(r_{0}-r_{i j}\right) \Theta\left(r_{0}-r_{i k}\right)$ are step functions, $\left\langle\theta_{i j k}\right\rangle$ is a constant, and $\theta_{i j k}$ is the angle between $r_{i j}$ and $r_{i k}$. The screenings in the Coulomb and in the charge-dipole interactions are introduced in order to avoid the long-range calculations in these interactions. The range of screening parameters was fixed in $\lambda=5.0 \AA$ and $\xi=3.75 \AA$, and the two-body potential is truncated at $r_{c}=7.5 \AA$. The interaction potential for $r<$ $r_{c}$ is shifted as usually $[19,20]$, in order to have the value and its first derivative continuous at the cutoff length. From other simulations using this type of potential [14, 15, 21] we took the exponents $\eta_{I n I n}, \eta_{I n S b}$, and $\eta_{S b S b}$ to be 7,9 , and 7 , respectively. The remaining constants were determined from the cohesive energy, elastic constant, bulk modulus, and melting temperature.

The molecular dynamics simulations were performed in the HPN ensemble (Parrinello-Rahman, which allows changes of the size and shape of the simulation box [1]), in a system consisting of 1000 particles $(500 \mathrm{In}+500 \mathrm{Sb})$. Initially the particles were arranged in a cubic zinc-blende structure at actual density, with zero external pressure. The system was then heated until temperature reaches $900 \mathrm{~K}$, when we start applying external pressure in a rate of 0.2 GPa per 50000 time steps, up to $6.0 \mathrm{GPa}$. For all applied pressures the temperature was kept constant at that value by scaling the velocity of particles every 100 time steps.

For each applied external pressure, the system was thermalized by 50000 time steps of $2.5 \mathrm{fs}$, and the phase space has been examined in order to provide two-body structural correlations through pair distribution function and coordination numbers, as well as three-body correlations through bond-angle distributions. It is known that the time scale for volume and shear fluctuations in the simulation ought to be on the order of a few vibrational periods; hence, they are by definition orders of magnitude smaller than experimental (seconds). Nevertheless, the phase transition may well occur very rapidly, as we discuss below. To determine the melting temperature, starting from the cubic zinc-blende structure, the system was heated at constant zero external pressure up to $1500 \mathrm{~K}$. For each temperature the system was allowed to relax for 50000 time steps.

The experimental values of the physical constants shown in the Table I were used to calibrate the interaction potential, that is, to adjust the parameters used in Eqs. (2) and (3). Once the results from the simulations reproduce well these values, the potential parameters are fixed to simulate all other properties. The values of the potential parameters for InSb are also displayed in Table II [4]. At zero pressure and zero kelvin the ground-state zinc-blende structure is stable.

TABLE I: Experimental (Refs. 29 and 30) and Molecular Dynamics values of the lattice parameter, elastic constants, bulk modulus, cohesive energy and melting temperature for InSb.

\begin{tabular}{lll}
\hline \hline & Experiment & Molecular dynamics \\
\hline \hline Lattice Parameter $(\stackrel{\mathscr{A}}{ })$ & 6.4794 & 6.4794 \\
Elastic Constants $(\mathrm{GPa})$ & & \\
$C_{11}$ & 65.76 & 65.7 \\
$C_{12}$ & 35.65 & 35.6 \\
$C_{44}$ & 29.83 & 25.2 \\
Bulk Modulus & 45.73 & 45.63 \\
Cohesive Energy (eV/N) & 2.80 & 2.795 \\
Melting Temperature $(\mathrm{K})$ & 800 & $1300 \pm 50$ \\
\hline \hline
\end{tabular}

TABLE II: Parameters used in the interaction potencial for InSb. $\lambda, \xi, r_{c}$ in angstrons..

\begin{tabular}{cccc}
\hline \hline & In-In & In-Sb & Sb-Sb \\
\hline \hline$H_{\alpha \beta}$ & $5.5933 \times 10^{-10}$ & $2.4105 \times 10^{-8}$ & $1.2022 \times 10^{-8}$ \\
$Z_{\alpha \beta}$ & $0.86825 e^{2}$ & $-0.86825 e^{2}$ & $0.86825 e^{2}$ \\
$D_{\alpha \beta}$ & 0 & $2.60476 e^{2}$ & $5.20951 e^{2}$ \\
$W_{\alpha \beta}$ & 0 & $14.539 \times 10^{-10}$ & 0 \\
$\eta_{\alpha \beta}$ & 7 & 9 & 7 \\
$\lambda=5.0$ & $\xi=3.75$ & $r_{\text {cut }}=7.5$ & $e$ = electron charge \\
\hline \hline
\end{tabular}




\section{Results and discussion for struc- tural properties}

To simulate the pressure effects on InSb, for each applied pressure, after the system has been very well thermalized, averages were taken over additional 20000 time steps. In this study the temperature of the system was set to be 900 K. In Fig. 1 we show the changes in the bond length, In$\mathrm{Sb}$ and first neighbors $\mathrm{Sb}-\mathrm{Sb}$ and In-In, as a function of the pressure. The increase in In-Sb bond length is followed by the increase of coordination number, which clearly show the structural transformation from a four-fold to sixfold coordinated orthorhombic structure under pressure.

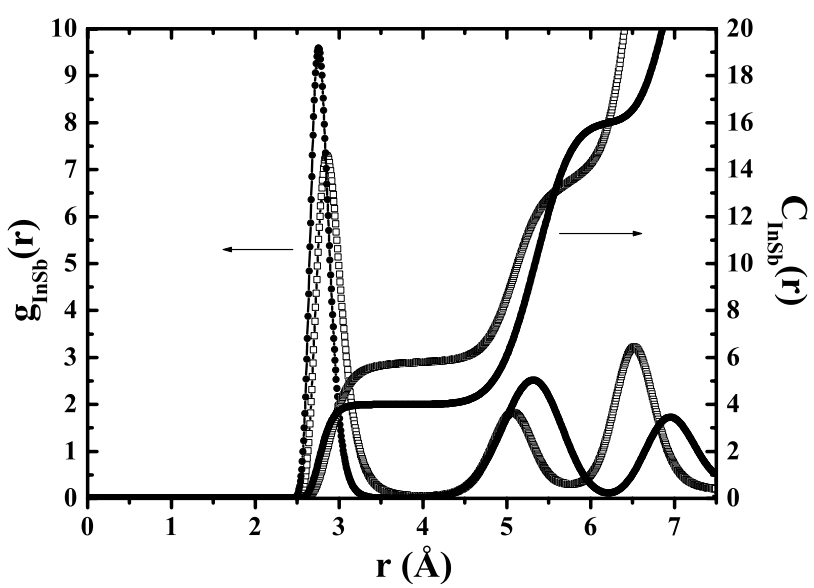

Figure 1. The In-Sb pair distribution function (left) and coordination number (right) for pressures just before and after the structural transformation. Full circles at $2.8 \mathrm{GPa}$ and open squares at 3.0 $\mathrm{GPa}$.

A comparison of experimental and MD results for the volume-pressure relationship for InSb is shown in Fig. 2 . An excellent agreement of the MD results, in a wide range of pressure can be observed. The volume reduction due to compression, just before the transition, at $2.8 \mathrm{GPa}$ is $0.925 V_{0}\left(V_{0}\right.$ is the volume at ambient pressure) which agrees very well with experimental results of $0.93 V_{0}$. The volume reduction due to the structural transformation was found to be $19.7 \%$, which also agrees very well with the experimental result reported by Nelmes et al.,[7] 19.5\%, and Yu et al.,[5] $19.3 \%$, but larger than that reported by Vanderborgh et al.[6] $17,1 \%$.

The three-body correlations were analyzed through the bond-angle distribution. Fig. 3 displays the Sb-In-Sb bondangle distribution at 2.8 and $3.0 \mathrm{GPa}$. At pressures below the structural transition the bond angle is peaked at $109^{\circ}$ (internal tetrahedral angle), and at the transition this angle moves to $90^{\circ}$ and $180^{\circ}$, characteristic of an orthorhombic phase. These results clearly demonstrate that the structural transformation from a tetrahedrally fourfold-coordinated structure goes to an octahedrally sixfold coordinated structure under pressure.

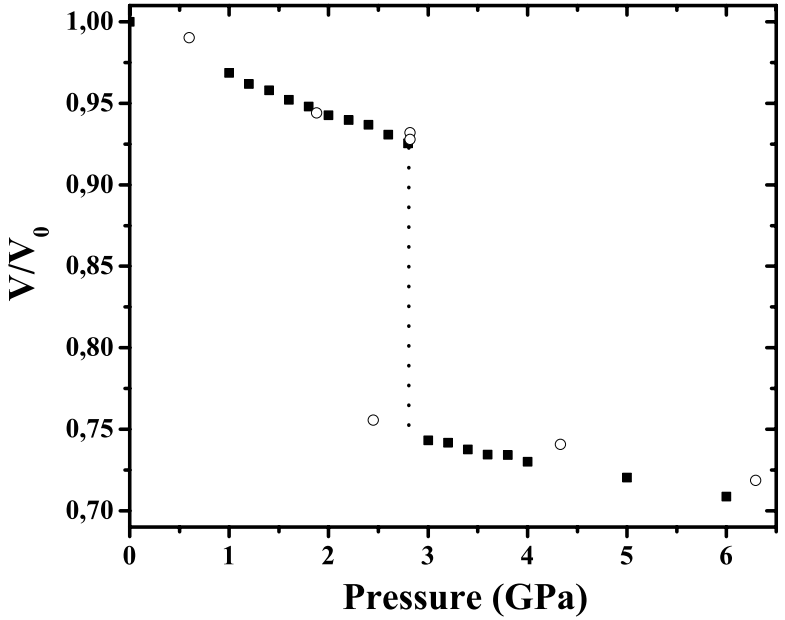

Figure 2. Changes of the reduced volume as a function of the hydrostatic pressure. Open circles from reference 5 and full squares from the molecular dynamics simulation. The dotted line indicates the structural phase transition.

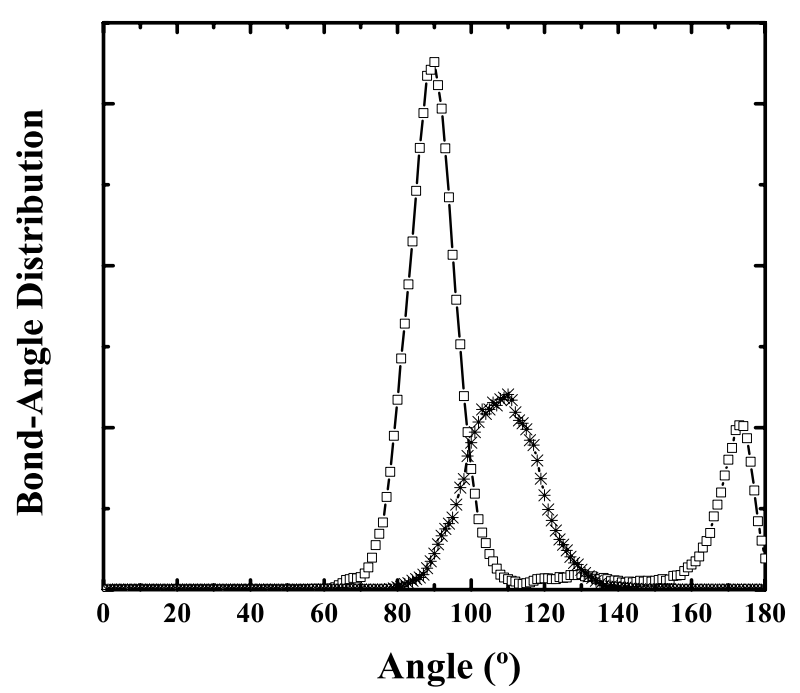

Figure 3. Sb-In-Sb bond angle at pressures just before and after the structural transformation. Open squares at $2.8 \mathrm{GPa}$ and stars at 3.0 GPa.

\section{Results and discussion for dynam- ics properties}

Here it will be focused the attention on the dynamical behavior of the ions in the crystalline phase as a function of the temperature, at normal pressure and also under high hydrostatic external pressure. From the atomic trajectory, furnished by the MD, it is possible to calculate all positional, angular and dynamical properties of the system. The velocity autocorrelation function $Z_{\alpha}(t)$ is defined as

$$
Z_{\alpha}(t)=\frac{\left\langle v_{i \alpha}(0) v_{i \alpha}(t)\right\rangle}{\left\langle v_{i \alpha}(0)^{2}\right\rangle},
$$


where $v_{i \alpha}(t)$ is the velocity of particle $i$ of type $\alpha$ at time $\mathrm{t}$ and $\langle>$ denotes an ensemble average as well as an average over all particles of type $\alpha$. The vibrational density of states $G(\omega)$ is obtained from the Fourier transform of the velocity-velocity correlation function

$$
G_{\alpha}(\omega)=\frac{6 N_{\alpha}}{\pi} \int_{0}^{\infty} Z_{\alpha}(t) \cos (\omega t) d t
$$

From the temperature dependence of the pair distribution function (PDF), which gives the bond distance between pair of atoms, it can be obtained the dynamical Debye-Waller factor and the thermal expansion coefficient.
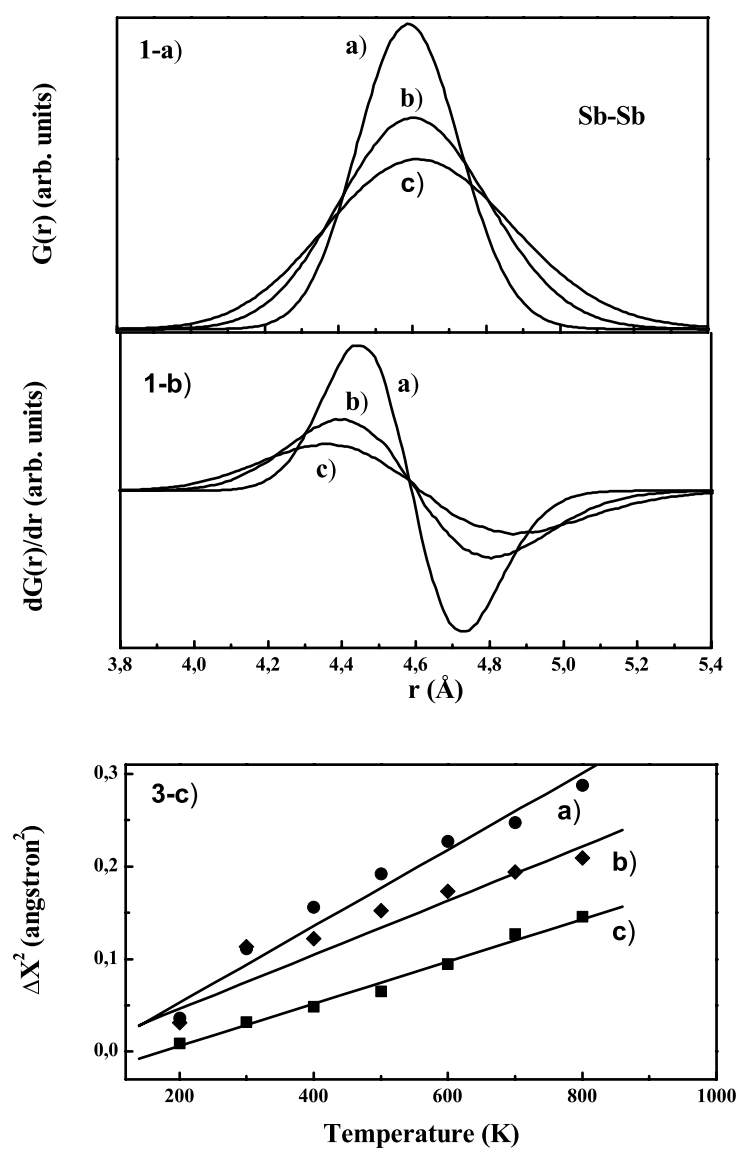

Figure 4. 4a I-a: first peak of the pair distribution function (PDF) of $\mathrm{Sb}$-Sb atoms in crystalline InSb for three different temperatures a) 300, b) 600 and c) $900 \mathrm{~K}$. I-b: first derivative of the PDF for the three temperatures. I-c: squared atomic amplitude oscillation for a) In-In, b) Sb-Sb and c) In-Sb as a function of the temperature.

Among the several static structural information that can be obtained from the pair distribution function (PDF) as bond length, atomic bond angle distributions, crystalline symmetry, and coordination numbers, it variation with the temperature and pressure gives information about structural phase transitions. Moreover, the variation with the temperature furnish directly the thermal expansion coefficient while the width of the pair distribution function gives the atomic oscillation amplitude, which permits to calculate the dynamical Debye-Waller factor. The data were obtained from the simulation in steps of $50 \mathrm{~K}$, from 200 to $1100 \mathrm{~K}$. In Fig. 4(a) it is showed the evolution of the first peak of the PDF of $\mathrm{Sb}-\mathrm{Sb}$ distance for three temperatures 300, 600, and 900 $\mathrm{K}$, at $0 \mathrm{GPa}$, whose maximum peak position gives the $\mathrm{Sb}$ $\mathrm{Sb}$ bond distance. From the zeros of the derivative of the first peak, displayed in Fig.4(b), it was obtained the thermal expansion coefficient for Sb-Sb, and similarly for In-In and InSb distances. The average values between 300 and $900 \mathrm{~K}$ are $\alpha_{\mathrm{Sb}-\mathrm{Sb}} \sim 10 \times 10^{-6}, \alpha_{\text {In-In }} \sim 14 \times 10^{-6}$ and $\alpha_{\text {In-Sb }} \sim 2 \times 10^{-6} \mathrm{~K}^{-1}$ for the cubic phase (at $0 \mathrm{GPa}$ ).

From the full width of the PDF it was obtained the atomic oscillation amplitude for $\mathrm{In}-\mathrm{Sb}$, In-In, and $\mathrm{Sb}-\mathrm{Sb}$ atoms $\Delta x$ as a function of the temperature, as displayed in Fig. 4(c). From a linear fit approximation, the temperature coefficient for $\left.\Delta x^{2},\left(\partial \Delta x^{2}\right) / \partial T\right)_{P}$, was obtained. The values are displayed in Table I. From these values, it can be determined the dynamical Debye-Waller factor by using the equation[22]

$$
D(T)=\exp [-2 M(T)]=\exp \left[\frac{-4 \pi^{2}(\Delta x)^{2}}{3 d^{2}}\right],
$$

where $d$ is the reticular spacing for the planes giving rise to the reflection under consideration.

Figure 5 displays the vibrational density of states $G(\omega)$ simulated at $0 \mathrm{GPa}$ and $300 \mathrm{~K}$, obtained by the Fourier transforms of the velocity-velocity correlation function and it comparison with the results obtained by the deformable bond model (DBM), [23] the experimental Raman spectrum[24] and with the phonon dispersion curve.[25] The result from MD fits very well the DBM and experimental results, reproducing the correct frequencies of the acoustical and optical bands. The lower values of the frequencies obtained from MD simulations can be attributed to the small number of particles (1000), since it sampled only around 80 $\%$ of the high values of the wave vector side of the Brioullin zone, while Raman scattering samples phonons at the center of the Brillouin zone (phonon wave vectors $k \sim 0$ ). As the dispersion relation of the optical modes presents decreasing frequencies with increasing phonon wave vectors, it is expected then lower values of the phonon frequencies from the simulations. Furthermore, the Raman scattering measurements from Ref. 8 were performed at low temperature ( 80 $\mathrm{K})$, which shifts the frequencies to higher values, standing out the differences. Despite the computational limitation, the frequency value of the maximum of the optical band is $170 \mathrm{~cm}^{-1}$, near $175 \mathrm{~cm}^{-1}$, the average frequency value of the optical band of InSb, at room temperature.[26] A discussion on the average optical frequency $\omega_{\text {op }}$ can be found in literature, which is defined by

$$
\omega_{\mathrm{op}}=\frac{1}{3 N} \sum_{k,, j} \omega_{j}(k),
$$

where $k$ belongs to the first Brillouin zone and $j$ are the optical branches. This equation describes the lattice dynamics as an Einstein approximation, where the optical modes are represented by $3 N$ oscillators vibrating at the same frequency $\omega_{\text {op. }} \cdot[27]$ 


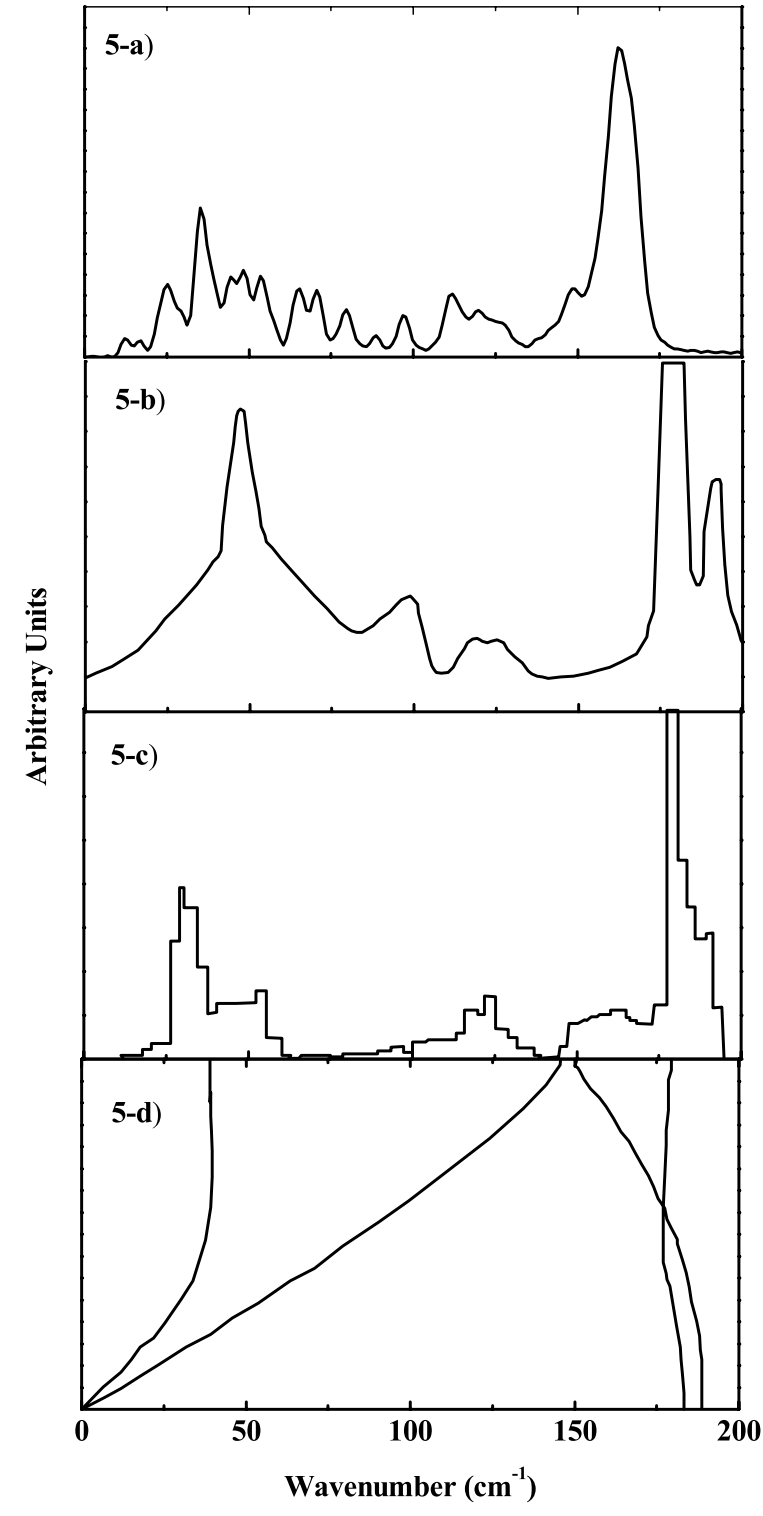

Figure 5. 5-a: vibrational density of states, G(w), from the Molecular Dynamics simulation at $0 \mathrm{GPa}$ and $300 \mathrm{~K}$. 5-b: Raman spectrum of InSb, reference 8. G(w) from the Deformable Bond Model, reference 7. 5-c: $\mathrm{G}(\mathrm{w})$ from the Deformable Bond Model, reference 7. 5-d: phonon dispersion curve for InSb, reference 9.

Figure 6(a) shows the effect of the temperature variation on $G(\omega)$, for 300,600 , and $900 \mathrm{~K}$. The simulations were performed from 200 to $1100 \mathrm{~K}$, in steps of $50 \mathrm{~K}$. Figure 6(b) displays the temperature dependence of the longitudinal (LO) and transverse (TO) optical phonon frequencies of InSb obtained by Raman scattering experiment[28] and the maximum of the optical band of the density of states, from the MD simulation. From a linear fitting approximation, the temperature frequency coefficients $\left(\partial \omega_{\mathrm{LO}} / \partial T\right)_{P}$, $\left(\partial \omega_{\mathrm{TO}} / \partial T\right)_{P}$ and $\left(\partial \omega_{\mathrm{op}} / \partial T\right)_{P}$ are $-0.026,-0.016$, and $0.025 \mathrm{~cm}^{-1} \mathrm{~K}^{-1}$, respectively.
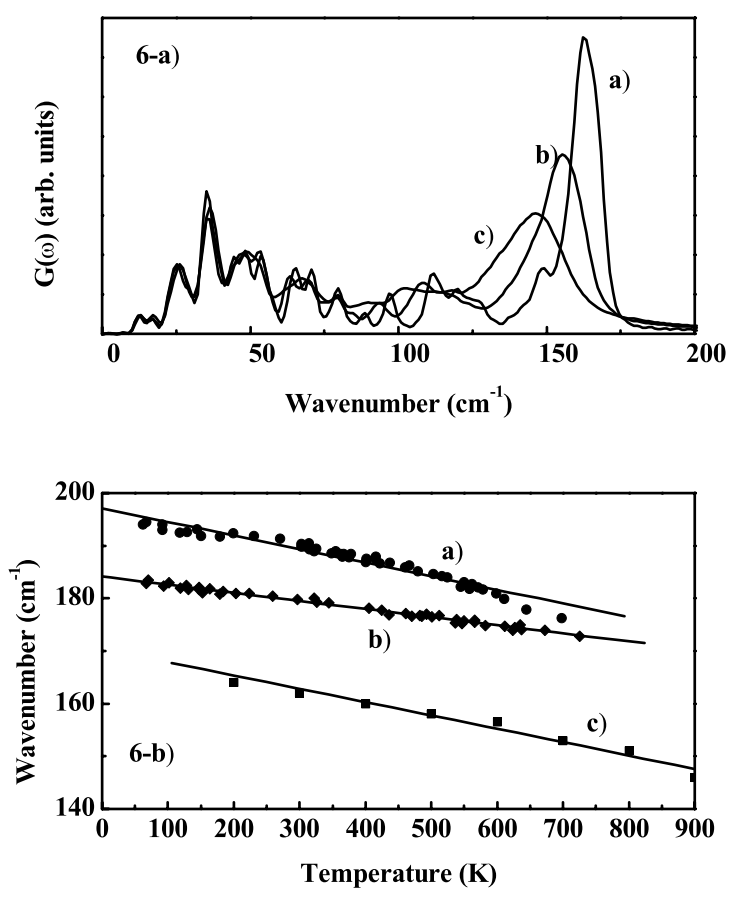

Figure 6. 6(a): Vibrational density of states $\mathrm{G}(\mathrm{w})$ at 300, 600 and 900 K. 6(b): a) and b) temperature dependence of the longitudinal and transverse optical phonon frequencies from Raman scattering, respectively and (c) temperature dependence of the maximum of $\mathrm{G}(\mathrm{w})$ in the optical range obtained by molecular dynamics simulation. The full lines are linear fitting approximations.
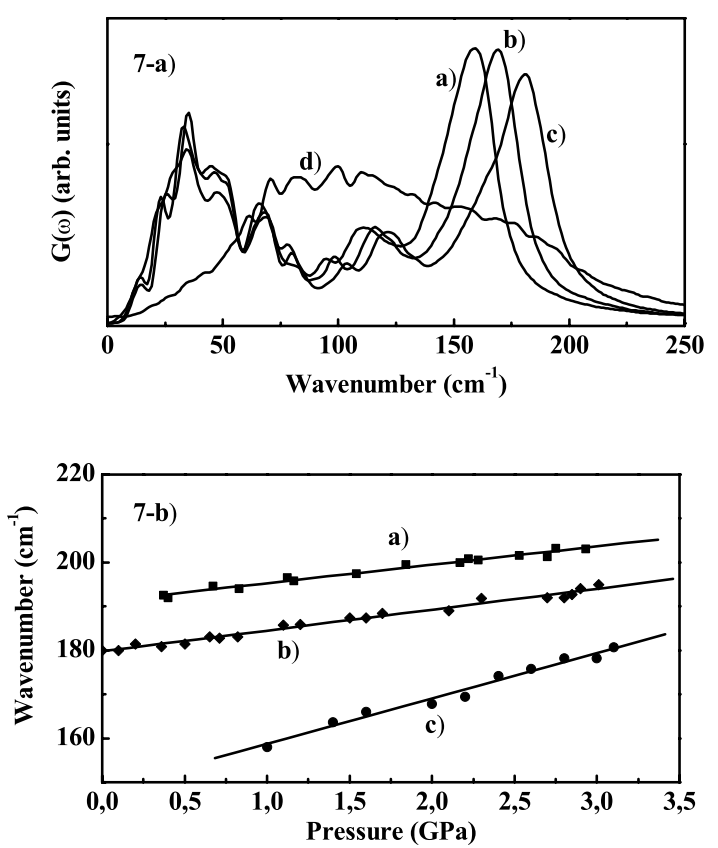

Figure 7. 7(a): Pressure dependence of $\mathrm{G}(\mathrm{w})$, from molecular dynamics simulation at (a) $1.0 \mathrm{GPa}$, (b) $2.0 \mathrm{GPa}$, (c) $3.0 \mathrm{GPa}$, and (d) 3.2 GPa. 7(b): a) and b) pressure dependence of the longitudinal and transverse optical phonon frequencies from Raman scattering, respectively from Ref. 29 and c) pressure dependence of the maximum of $G(w)$ in the optical range obtained by molecular dynamics simulation. The full lines are linear fitting approximations. 
TABLE III: Values of the physical constants obtained by Molecular Dynamics simulations and from the literature. $\alpha_{S b-S b}, \alpha_{I n-I n}$, $\alpha_{I n-S b}$, are the mean thermal expansion coefficient between 300 and $\left.900 \mathrm{~K} .\left(\partial \Delta x^{2}\right) / \partial T\right)_{P}$, is the temperature coefficient for the squared atomic amplitude oscilation. $\omega_{o p}$, is the maximum of the optical band and $\left(\partial \omega_{o p} / \partial T\right)_{P}$, and $\left(\partial \omega_{o p} / \partial P\right)_{T}$, are it temperature and pressure coefficient, respectively. $\left(\partial \omega_{L O} / \partial T\right)_{P},\left(\partial \omega_{T O} / \partial T\right)_{P},\left(\partial \omega_{L O} / \partial P\right)_{T},\left(\partial \omega_{T O} / \partial P\right)_{T}$, are the temperature and pressure frequency coefficients for the longitudinal and transverse optical phonons, respectively.

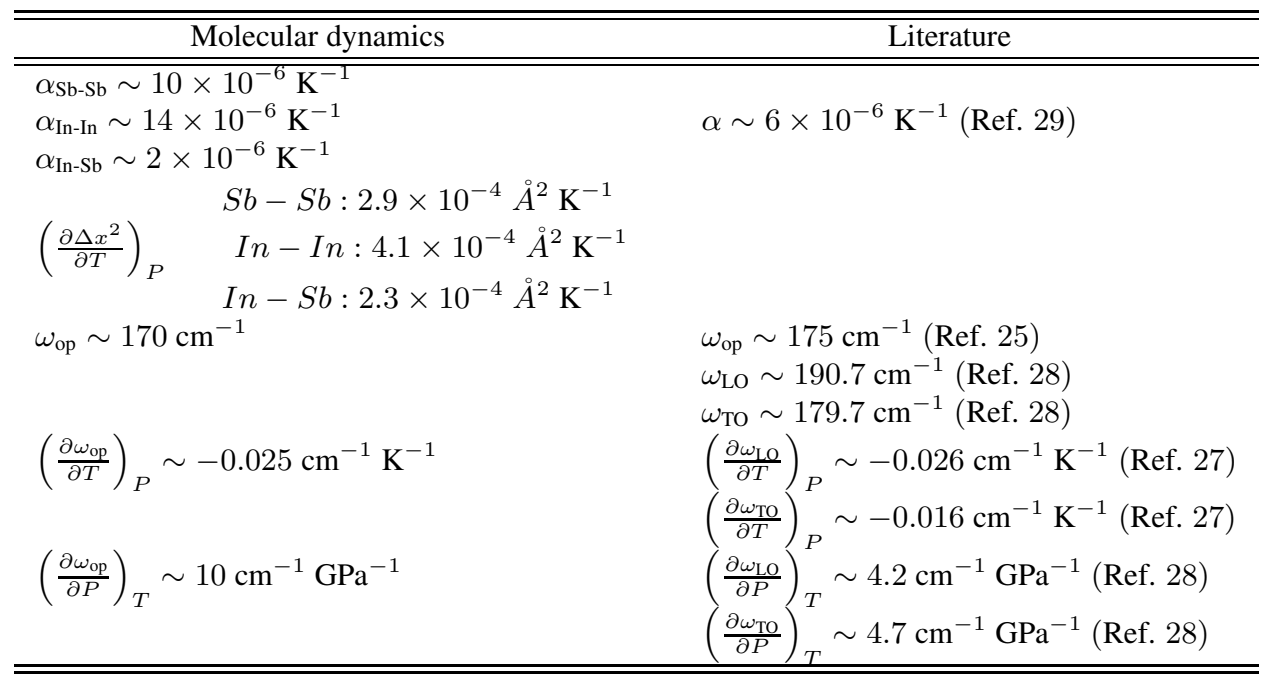

Figure 7(a) shows the dependence of the $G(\omega)$ with the hydrostatic pressure. There are two main effects of the pressure on $G(\omega)$ : (i) an anharmonic frequency shift to high frequencies up to $3.0 \mathrm{GPa}$ and (ii) a structural cubic to orthorhombic phase transition, denounced by the dramatic change in $G(\omega)$ at about 3.2 GPa. Figure 7(b) displays the pressure dependence of the longitudinal optical phonon of crystalline InSb from Raman scattering[29] and of the maximum of $G(\omega)$. From a linear fitting approximation, the pressure frequency coefficients $\left(\partial \omega_{\mathrm{LO}} / \partial P\right)_{T}$, $\left(\partial \omega_{\mathrm{TO}} / \partial P\right)_{T}$, and $\left(\partial \omega_{\mathrm{op}} / \partial P\right)_{T}$ are $4.2,4.7$, and $10 \mathrm{~cm}^{-1}$ $\mathrm{GPa}^{-1}$, respectively. Finally, Table III summarizes all numerical values obtained from the present simulation, showing also the experimental values disponible in the literature.

\section{Conclusions}

In conclusion, isoenthalpic-isobaric molecular dynamics simulations to study the pressure and temperature influence on structural and dynamical properties of InSb was successfully performed using the effective potential which takes into account two and three-body interactions. The zincblende to orthorhombic structural transformation is very well described, in excelent agreement with experimental observation. The calculated volume change, before and after transformation, are also in excellent agreement with those observed in experiments. Furthermore, structural parameters such as bond length and coordination number are correctly reproduced.

From the pair distribution function, the thermal expansion coefficient was obtained, in excellent accord with the experimental value. Furthermore, the temperature dependence of the dynamical Debye-Waller factor was also obtained. From the vibrational density of states, the temper- ature and pressure phonon anharmonicity was correctly described, in excellent accord with results from Raman scattering. As the interatomic potential describes very well static and dynamic properties of InSb, it can be used to simulate and preview new properties in several different experimental conditions.

\section{Acknowledgments}

This work was parcially supported by Fundação de Amparo à Pesquisa do Estado de São Paulo - FAPESP and Conselho Nacional de Desenvolvimento Científico e Tecnológico - CNPq.

\section{References}

[1] M. Parrinello and A. Rahman, Phys. Rev. Lett. 45, 1196 (1980); M. Parrinello and A. Rahman, J. Appl. Phys. 52, 7182 (1981).

[2] K. Asaumi, O. Shimomura, and S. Minomura, J. Phys. Soc. Jap. 41, 1630 (1976)

[3] O. Shimomura, K. Asaumi, N. Sakay, and S. Minomura, Philos. Mag. 34, 839 (1976).

[4] S.C. Costa, P.S. Pizani, and J.P. Rino, Phys. Rev. B, 66, 214111 (2002).

[5] S. C. Yu, I. L. Spain, and E. F. Skelton, Solid State Commun. 25, 49 (1978)

[6] C. A. Vanderborgh, Y. K. Vohra, and A. L. Ruoff, Phys. Rev. B 40, 12450 (1989).

[7] R. J. Nelmes, M. I. McMahon, P. D. Hatton, J. Crain, and R. O. Piltz, Phys. Rev. B 47, 35 (1993).

[8] R. J. Nelmes and M. I. McMahon, Phys. Rev. Lett. 77, 663 (1996). 
[9] S. B. Zang and M. L. Cohen, Phys. Rev B 35, 7604 (1987).

[10] G. Y. Guo, J. Crain, P. Blaha, and W. M. Temmermen, Phys. Rev. B 47, 4841 (1993).

[11] A. A. Kelsey and G. J. Ackland, J. Phys. Condens. Matter 12, 7161 (2000).

[12] F. H. Stillinger and T. A. Weber, Phys. Rev. B 31, 5262 (1985).

[13] S. Erkoç Phys. Reports. 278, 79 (1997) (in this paper several empirical interaction potentials are discussed).

[14] F. Shimojo, I. Ebbsjo, R. K. Kalia, A. Nakano, J. P. Rino, and P. Vashishta, Phys. Rev. Lett. 84, 3338 (2000).

[15] I. Ebbsjo, R. K. Kalia, A. Nakano, J. P. Rino, and P. Vashishta, J. Appl. Phys. 87, 7708 (2000).

[16] W. Li, R. K. Kalia and P. Vashishta, Phys. Rev. Lett. 77, 2241 (1996).

[17] R. K. Kalia, A. Nakano, K. Tsuruta, and P. Vashishta, Phys. Rev. Lett. 78, 689 (1997).

[18] R. K. Kalia, A. Nakano, A. Omeltchenko, K. Tsuruta, and P. Vashishta, Phys. Rev. Lett. 78, 2144 (1997).

[19] M. P. Allen and D. J. Tildesley, "Computer Simulation of Liquids", Clarendon Press - Oxford (1997).

[20] A. Nakano, R. K. Kalia, and P. Vashishta, J. Non-Crsyt. Solids 171, 157 (1994).

[21] J. P. Rino, A. Chatterjee, I. Ebbsjö, R. K. Kalia, A. Nakano, F. Shoimojo, and P. Vashishta, Phys. Rev. B 65, 195206-1 (2002).
[22] A. Guinier, X-Ray Diffraction in Crystals, Imperfect Crystals and amorphous Bodies (W. H. Freeman and Company, San Francisco, 1963), Chap. 7.

[23] K. Kunc, M. Balkanski and M. A. Nusimovici, Phys. Status Solidi (b) 72, 229 (1975).

[24] W. Kiefer, W. Richter and M. Cardona, Phys. Rev. B, 12, 2346 (1975).

[25] D. L. Price, J. M. Rowe and R. M. Nicklow, Phys. Rev. B, 3, 1268 (1971)

[26] G. Landa, Spectrometrie Raman des Materiaux III-V Desordonnes, $\mathrm{Ph}$. D. Thesis, Université Paul Sabatier, Toulouse, France (1990).

[27] R. Carles, G. Landa and J. B. Renucci, Solid State Commun. 53, 179 (1985).

[28] E. Liarokapis and E. Anastassakis, Phys. Rev. B 30, 2270 (1984).

[29] K. Aoki, E. Anastassakis and M. Cardona, Phys. Rev. B, 30, 681 (1984).

[30] Numerical Data and Functional Relationships in Science and Technology, edited by O. Madelung, M. Schulz and H. Weiss, Landolt-Börnstein, New Series, Group III, Vol. 17a, Pt. 327 (Springer-Verlag, Berlin, 1982).

[31] W. A. Harrison, Electronic Structure and the Properties of Solids (W. H. Freeman and Company, San Francisco, 1980). 